\title{
REPLY
}

\section{Response to Cardiovascular control during head-up tilt test in spinal cord injury patients}

\author{
Spinal Cord (2011) 49, 674; doi:10.1038/sc.2010.154; \\ published online 2 November 2010
}

Thank you for letting me review this Letter to the Editor, ${ }^{1}$ which refers to our paper. ${ }^{2}$ I would like to thank the author for the interest in our study.

The letter suggests that the comparison of findings in the article in support of the suggested hypothesis is irrelevant, and cites other findings that supposedly support another unproven and not entirely clear hypothesis. The comparison is relevant, however, and the additional information in the letter is not new, and neither supports nor refutes our hypothesis. The findings presented in our paper contradict a common conception and suggest a mechanism that may explain the findings. Additional propositions and citations that divert the attention to another unproven mechanism, in which the author apparently believes, do not necessarily strengthen the argument of our paper.

Our paper addresses the possibility, presented in the letter, that the $T_{1}-T_{5}$ sympathetic outflow and the Vagus nerve are the only efferent components of the autonomic nervous system involved in maintaining blood pressure during headup tilt in $\mathrm{T}_{4}-\mathrm{T}_{6}$ paraplegia. But our paper also presents the finding that in the same patients with $\mathrm{T}_{4}-\mathrm{T}_{6}$ paraplegia in whom blood pressure was maintained after head-up tilt, blood pressure dropped after the meal, despite the fact that these components were intact. This implies that the mechanism working through these components may not be sufficient to compensate for the blood pressure drop in these patients, and that an additional mechanism may be required to maintain their blood pressure. The letter maintains that 'it is not relevant to compare cardiovascular reactions after a meal to those obtained during HUT,' but there is no evidence to support this claim. The letter mentions additional mechanisms that may be involved in the responses to the meal and head-up tilt, but it does not show how these mechanisms can explain the differences between responses to the meal and the head-up tilt. None of the findings described in the letter serve as proof of the mechanism in which the correspondent appears to believe.

I therefore recommend that the author or other interested researchers conduct a study that confirms or refutes our hypothesis.

\section{Conflict of interest}

The author declares no conflict of interest.

\author{
A Catz \\ Department IV, Spinal Rehabilitation, Loewenstein \\ Rehabilitation Hospital, Raanana, Israel \\ E-mail: amcatz@post.tau.ac.il
}

\section{References}

1 Prévinaire JG, Soler JM. Cardiovascular control during head-up tilt test in spinal cord injury patients. Letter to the editor. Spinal Cord 2011; 49: 673 .

2 Bluvshtein V, Korczyn AD, Akselrod S, Pinhas I, Gelernter I, Catz A. Hemodynamic responses to head-up tilt after spinal cord injury support a role for the mid-thoracic spinal cord in cardiovascular regulation. Spinal Cord 2011; 49: 251-256. 